Rev. salud pública. 14 sup (2): 3-14, 2012

Artículos Originales/Original Articles

\title{
Gestión de gobiernos locales en la calidad de la atención de la salud para niños
}

\section{Local government management regarding the quality of children's healthcare}

\author{
Aldemar Bautista-Otero' y Juan C. Garcia-Ubaque ${ }^{2}$ \\ 1 Facultad de Ciencias Económicas, Universidad Nacional de Colombia. Bogotá, Colombia. abautistao \\ @unal.edu.co \\ 2 Facultad de Enfermería, Centro de Investigaciones para el Desarrollo (CID). Universidad Nacional \\ de Colombia. Bogotá, Colombia. jcgarciau@unal.edu.co
}

Recibido 25 Mayo 2011/Enviado para Modificación 8 Agosto 2011/Aceptado 3 Noviembre 2011

\section{RESUMEN}

Objetivo Evaluar el componente de calidad de los programas de atención en salud dirigidos a niños, niñas y adolescentes establecidos por los gobiernos locales de Colombia en cumplimiento de las normas legales.

Métodos Estudio descriptivo en el que se evaluó la evidencia de rectoría de los sistemas de calidad y del cumplimiento de los atributos de accesibilidad, oportunidad, seguridad, continuidad y pertinencia como indicadores de la calidad en la prestación de servicios.

Resultado No se encontró evidencia que demuestre que los gobiernos locales examinados desarrollen programas de salud orientados a la niñez que sean específicos a su realidad epidemiológica. Las actividades que se ejecutan corresponden al mínimo legal y no son evaluados en su componente de calidad, por tanto no hay un enfoque de mejoramiento en los mismos.

Conclusión Se proponen estos resultados como línea base y dado que la función de los gobiernos locales, en materia de calidad en salud, va desde la planeación, pasando por la rectoría y el control de la calidad de los prestadores, se hace inaplazable la implementación de programas de gestión de la calidad antes que nuevos programas de atención.

Palabras Clave: Calidad de la atención de salud, regulación y fiscalización en salud, servicios de salud del niño, planes y programas de salud, gobierno local. (fuente: DeCS, BIREME).

\section{ABSTRACT}

Objective Assessing the quality of healthcare programs aimed at children and adolescents set by Colombian local governments in compliance with current legal standards. 
Methods This was a descriptive study which evaluated the evidence regarding surveillance of quality systems and compliance with accessibility, opportunity, safety, continuity and relevance as indicators of quality concerning service delivery. Results No evidence was found showing that the local governments so examined were developing child-orientated health programs specifically addressing epidemiological reality. Ongoing activities complied with minimum legal requirements and were not being evaluated regarding their quality, meaning that an approach aimed at improvement was not being adopted.

Conclusion The above results are thus proposed as a working baseline and, given that the role of local government in terms of healthcare quality ranges from planning, to surveillance and quality control concerning providers, quality management programs must be implemented before new healthcare programs are introduced.

Key Words: Quality healthcare, health regulation and control, children's health services, Healthy people programs, local government (source: MeSH, NLM).

$\mathrm{C}$ olombia presenta un patrón mixto de morbimortalidad (1); en el que aún siguen presentes patologías típicas de países en desarrollo carenciales y las alteraciones perinatales y asociadas a la maternidad; al igual que dolencias más asociadas a países desarrollados, como el cáncer, las alteraciones del aparato circulatorio, los accidentes de carretera, las enfermedades mentales, la dependencia de drogas y el alcoholismo y de manera particular los problemas asociados a las diferentes clases de violencia (2). Esto implica una gran complejidad para el sistema de salud y obliga a adoptar estrategias que minimicen la misma, entre las cuales la calidad de la atención de salud resulta relevante (3), así como el enfoque en poblaciones prioritarias (4), entre las cuales se destacan los niños, niñas $\mathrm{y}$ adolescentes (NNA); siendo claro que es esencial medir la efectividad y el impacto de los programas, ya que de nada servirían los mismos si no cuentan con un nivel de avance que les permitan ser exitosos (5).

El sistema general de seguridad social en salud (SGSSS) lo conforman cinco grupos de agentes: a. Los organismos estatales de regulación y administración así como los de inspección, vigilancia y control (IVC); b. los organismos de administración y financiación o entidades administradoras de planes de beneficios; c. las instituciones prestadoras de servicios de salud, públicas, mixtas o privadas; d. las entidades de salud adscritas al ministerio de la protección social; e. los empleadores, los trabajadores, los pensionados, los beneficiarios de estos y el resto de la población, así 
como las organizaciones en las que se agrupan. Cada uno de ellos tiene, de acuerdo con la ley, un rol específico que busca lograr un sistema de salud equitativo, obligatorio, con atención integral, libre escogencia, autonomía de las instituciones, descentralización, participación social, y concertación, logros que se pretenden alcanzar mediante unos planes de beneficio específicos (6). No obstante, el desarrollo del sistema ha evidenciado que con frecuencia los intereses enfrentados de estos agentes, así como la debilidad de las instituciones rectoras, dificultan el logro de estos propósitos, por lo que se ha recomendado un fortalecimiento de la función estatal, en particular de los aspectos de IVC (7).

La defensa y garantía de los derechos de los niños y adolescentes es una tarea promovida, desde su creación en 1946, por la UNICEF (Fondo Internacional de Emergencias de la Infancia de las Naciones Unidas), que ha dado origen entre otros a la declaración de los derechos del niño en 1953 y a la convención sobre el mismo tema en 1989 (8).

En Colombia la constitución política establece que los derechos del niño prevalecen sobre los demás (9), lo que ha generado legislación que busca materializar esta concepción mediante la asignación de obligaciones al sector público y privado (10), entre otras áreas en el campo de la salud; En este sentido la Superintendencia Nacional de Salud, como máximo ente de control del sector salud (11), realizó auditorías a las entidades territoriales para verificar el cumplimiento de la Ley (12). A partir la información recolectada en este proceso se revisó la misma, con el fin de verificar que los mecanismos que vigilan que la prestación de los servicios de atención en salud para NNA se realicen en condiciones de disponibilidad, accesibilidad, aceptabilidad y estándares de calidad, tanto en las fases de promoción, prevención, diagnóstico, tratamiento, como en las de rehabilitación (13).

Esta información resulta relevante dado lo numeroso y vulnerable que es este grupo poblacional (14) y la importancia de evaluar la calidad de los programas de atención en salud (15).

\section{MATERIALES Y MÉTODOS}

Se realizó un estudio descriptivo de corte transversal, en el que para realizar la evaluación, se revisaron los informes de la superintendencia de salud 
desde dos aspectos: por una parte, la gestión en calidad y, por otra parte, los programas de atención a la infancia en el contexto de los gobiernos locales y departamentales colombianos durante la vigencia 2007 a 2009.

La metodología utilizada para la recolección de información se basó en tres (3) elementos: los formatos de recolección de información, las herramientas de definición de alcances y soporte normativo y los instructivos o indicaciones para la utilización de los anteriores.

El formato de recolección de información precisó cinco aspectos: a. El requerimiento o pregunta; b. El modo de verificación de la respuesta; c. La verificación realizada; d. El estado de desarrollo del requerimiento considerado en cinco posibilidades o fases: documentación, normalización, socialización, implementación o evaluación, y; e. Las observaciones al proceso.

Los requerimientos o preguntas se centraron en los dos tópicos mencionados, los cuales se corresponden con los grandes roles de los gobiernos locales en este campo: el papel rector de los sistemas de calidad y la vigilancia del cumplimiento de los atributos de calidad en la prestación de servicios. En el primero se verifico la existencia de diagnóstico de la situación de la calidad de la prestación de los servicios de salud y de planes y programas para el mejoramiento de la calidad que incluyesen como mínimo asistencia técnica y asesoría, así como el registro de prestadores públicos y privados y las acciones de vigilancia y control al sistema único de habilitación de prestadores y la divulgación de la normatividad encaminada al mejoramiento de la salud, en particular asesoría frente a la implementación del programa de auditoría para el mejoramiento de la calidad de la atención en salud y el desarrollo de actividades de sensibilización y capacitación frente al desarrollo del sistema único de acreditación. Mientras que en el segundo la indagación se centró en los atributos de accesibilidad, oportunidad, seguridad, pertinencia y continuidad.

La accesibilidad definida como la posibilidad que tienen los Niños, Niñas y Adolescentes (NNA) de utilizar los servicios de salud que le garantiza el SGSSS. La oportunidad en términos de obtener los servicios que requieren, sin que se presenten retrasos que pongan en riesgo su vida o su salud, este atributo se relaciona con la organización de la oferta de servicios en relación con la demanda y con el nivel de coordinación institucional 
para gestionar el acceso a los servicios. La seguridad como el conjunto de elementos estructurales, procesos, instrumentos y metodologías basadas en evidencias científicamente probadas que propenden por minimizar el riesgo de sufrir un evento adverso en el proceso de atención de salud o de mitigar sus consecuencias. La pertinencia definida como el grado en el cual las niñas y los niños obtienen los servicios que requieren, con la mejor utilización de los recursos de acuerdo con la evidencia científica y sus efectos secundarios son menores que los beneficios potenciales. Y finalmente la continuidad entendida como el grado en el cual los infantes reciben las intervenciones requeridas, mediante una secuencia lógica y racional de actividades, basada en el conocimiento científico (16). Al final se califica el desempeño del gobierno local, en materia de calidad en salud, como incipiente (I), regular (R), aceptable (A), suficiente (S) o excelente (E), según el grado de cumplimiento de sus funciones.

Para el diligenciamiento de la herramienta se efectuó una homogenización de términos a través de la herramienta "instructivo para el diligenciamiento de los instrumentos de recolección de información". La limitación de esta investigación está dada por el estudio que le dio origen, puesto que se limita a los entes territoriales incluidos en los convenios interadministrativos.

\section{RESULTADOS}

La población colombiana de niños, niñas y adolescentes, es decir la población menor de 18 años, alcanza el 36,8 \%, lo que significa que se está realizando un estudio que incluye más de un tercio de la población colombiana. La distribución por género muestra un perfecto equilibrio entre niños y niñas.

Los resultados combinan tres aspectos: La caracterización general de la población infantil, la existencia de programas de atención a NNA en el contexto de los entes territoriales y la vigilancia de los atributos de calidad, tanto en programas generales como específicos, durante las vigencias 2007 a 2009.

El Cuadro 1 evidencia la situación de la gestión de programas de salud para NNA por parte de los gobiernos locales examinados, como se aprecia en la misma, ninguno supera la calificación de aceptable ya que en términos generales no se han creado programas específicos para dar cumplimiento a 
ley de infancia y se continua dando una cobertura similar a la que se venía realizando antes de la promulgación de la ley, esencialmente por medio de los mismos programas que dan cumplimiento al plan nacional de salud pública.

Cuadro 1. Situación de la gestión de gobiernos locales colombianos en la calidad de la atención de la salud para niños 2010

\begin{tabular}{|c|c|c|c|}
\hline $\mathrm{GL}$ & Acciones desarrolladas & Nivel & Observación \\
\hline 1 & $\begin{array}{c}\text { Registro de prestadores; IVC } \\
\text { habilitación; Divulgación; Valora dos } \\
\text { atributos de calidad }\end{array}$ & A & Programas deficientes \\
\hline 2 & Registro de prestadores & I & Acciones generales y aisladas \\
\hline 3 & Registro de prestadores & 1 & $\begin{array}{l}\text { Balance discutible en distribución de } \\
\text { recursos }\end{array}$ \\
\hline 4 & Registro de prestadores & I & Acciones generales y aisladas \\
\hline 5 & Registro de prestadores & I & Acciones generales y aisladas \\
\hline 6 & $\begin{array}{l}\text { Registro de prestadores; IVC } \\
\text { habilitación }\end{array}$ & $\mathrm{R}$ & $\begin{array}{l}\text { Balance discutible en distribución de } \\
\text { recursos }\end{array}$ \\
\hline 7 & Registro de prestadores; Divulgación & $\mathrm{R}$ & $\begin{array}{c}\text { Desconocimiento de especificidad } \\
\text { NNA }\end{array}$ \\
\hline 8 & $\begin{array}{c}\text { No hay evidencia de existencia de } \\
\text { ningún mecanismo }\end{array}$ & I & $\begin{array}{c}\text { Importante cobertura de servicios } \\
\text { inespecíficos }\end{array}$ \\
\hline 9 & Registro de prestadores & $\mathrm{R}$ & $\begin{array}{l}\text { Promueven derechos de NNA con } \\
\text { acciones generales }\end{array}$ \\
\hline 10 & Registro de prestadores; Divulgación & B & $\begin{array}{c}\text { Medidas parciales de protección a } \\
\text { NNA }\end{array}$ \\
\hline 11 & Registro de prestadores & 1 & Acciones generales y aisladas \\
\hline 12 & Registro de prestadores & I & $\begin{array}{c}\text { Desconocimiento de especificidad } \\
\text { NNA }\end{array}$ \\
\hline 13 & Registro de prestadores; Divulgación & $\mathrm{R}$ & $\begin{array}{l}\text { Situación alarmante de NNA, } \\
\text { particularmente en primera infancia }\end{array}$ \\
\hline 14 & Registro de prestadores & $\mathrm{R}$ & $\begin{array}{c}\text { Desconocimiento de especificidad } \\
\text { NNA }\end{array}$ \\
\hline 15 & Registro de prestadores & 1 & $\begin{array}{l}\text { Incumplimiento NNA, deficiente } \\
\text { ejecución del plan nacional de salud } \\
\text { publica }\end{array}$ \\
\hline 16 & Registro de prestadores; Divulgación & $\mathrm{R}$ & $\begin{array}{c}\text { Desconocimiento de especificidad } \\
\text { NNA }\end{array}$ \\
\hline 17 & Registro de prestadores; Divulgación & $\mathrm{R}$ & $\begin{array}{c}\text { Balance discutible en distribución de } \\
\text { recursos }\end{array}$ \\
\hline 18 & Registro de prestadores & I & $\begin{array}{c}\text { Incumplimiento NNA, deficiente } \\
\text { ejecución del plan nacional de salud } \\
\text { publica }\end{array}$ \\
\hline 19 & Registro de prestadores; Divulgación & $\mathrm{R}$ & $\begin{array}{l}\text { Incumplimiento NNA, limitada } \\
\text { ejecución del plan nacional de salud } \\
\text { publica }\end{array}$ \\
\hline 20 & $\begin{array}{l}\text { Registro de prestadores; IVC } \\
\text { habilitación; Divulgación }\end{array}$ & A & $\begin{array}{l}\text { Algunas acciones especificas con } \\
\text { NNA }\end{array}$ \\
\hline 21 & Registro de prestadores & $\mathrm{R}$ & $\begin{array}{c}\text { Desconocimiento de especificidad } \\
\text { NNA }\end{array}$ \\
\hline
\end{tabular}




\begin{tabular}{|c|c|c|c|}
\hline GL & Acciones desarrolladas & Nivel & Observación \\
\hline 22 & Registro de prestadores & I & $\begin{array}{c}\text { Incumplimiento NNA, limitada } \\
\text { ejecución del plan nacional de salud } \\
\text { publica }\end{array}$ \\
\hline 23 & Registro de prestadores & $\mathrm{R}$ & $\begin{array}{c}\text { Algunas acciones específicas con } \\
\text { NNA }\end{array}$ \\
\hline 24 & Registro de prestadores; Divulgación & $\mathrm{R}$ & $\begin{array}{l}\text { Algunas acciones específicas con } \\
\text { NNA y enfoque etnocultural }\end{array}$ \\
\hline 25 & $\begin{array}{c}\text { Registro de prestadores; IVC } \\
\text { habilitación; Divulgación; PAMEC } \\
\text { incipiente }\end{array}$ & A & Acciones generales y aisladas \\
\hline 26 & Registro de prestadores; Divulgación & $\mathrm{R}$ & Acciones generales y aisladas \\
\hline 27 & Registro de prestadores; Divulgación & $\mathrm{R}$ & $\begin{array}{l}\text { Incumplimiento NNA, deficiente } \\
\text { ejecución del plan nacional de salud } \\
\text { publica }\end{array}$ \\
\hline 28 & $\begin{array}{l}\text { Registro de prestadores; valora dos } \\
\text { atributos de calidad }\end{array}$ & $\mathrm{R}$ & Acciones generales y aisladas \\
\hline 29 & $\begin{array}{c}\text { Registro de prestadores; PAMEC } \\
\text { incipiente; valora dos atributos de } \\
\text { calidad }\end{array}$ & $\mathrm{R}$ & $\begin{array}{l}\text { Algunas acciones específicas con } \\
\text { NNA, pero sin enfoque etnocultural }\end{array}$ \\
\hline 30 & Registro de prestadores & I & $\begin{array}{l}\text { Incumplimiento NNA, deficiente } \\
\text { ejecución del plan nacional de salud } \\
\text { pública }\end{array}$ \\
\hline 31 & Registro de prestadores & $\mathrm{R}$ & $\begin{array}{c}\text { Algunas acciones específicas con } \\
\text { NNA }\end{array}$ \\
\hline
\end{tabular}

Se evidencia que la mayor parte de los gobiernos locales evaluados no cuenta con un programa sistemático de gestión de la calidad, ni específico para la población de estudio, ni general para el sistema de seguridad social en salud, en este aspecto la información recogida permite señalar que aunque muchos brindan algún nivel de asistencia técnica para implementar la Auditoria para el Mejoramiento de la Calidad de la Atención de Salud en los Prestadores de Servicios de Salud de su jurisdicción, dichas actividades no son estandarizadas ni sistemáticas, por lo que están lejos de constituirse en un programa e igualmente que aunque hay unos logros en el sistema único de acreditación estos son limitados y obedecen principalmente a programas nacionales de apoyo y a tareas individuales de los prestadores, antes que ha esfuerzos territoriales.

La explicación que dichos gobiernos dan ante esta situación es que hay mucha dispersión geográfica de los vigilados y que tienen una gran limitación de recurso humano y que su enfoque prioritario se encuentra en este momento en garantizar algún nivel de servicio para la población no afiliada al SGSSS y en segundo lugar para los procesos propios del régimen subsidiado en salud de su territorio, no obstante lo cual es importante señalar que la formulación, ejecución y evaluación de planes, programas y 
proyectos de mejoramiento de la calidad en salud, está en armonía con las políticas y disposiciones del orden nacional y departamental.

Frente a la evaluación específica de atributos de calidad de los programas existe un nivel básico de seguimiento a la accesibilidad de los servicios de salud, pero este es consecuencia principal de las actividades generales de cumplimiento del sistema general de seguridad social en salud y por tanto resultan ser servicios poco específicos para el requerimiento de los NNA; Solo tres de los gobiernos locales valoran al menos dos de estos atributos y ninguno valora tres o más, con lo que el seguimiento resulta muy deficiente, ya que no se lleva registro sistemático de la situación actual de oportunidad, continuidad o seguridad en la prestación de los servicios de salud para NNA en las diferentes zonas de influencia de los gobiernos locales examinados.

La actividad rectora de los entes territoriales está limitada al desarrollo del Sistema Obligatorio de Calidad en Salud de las IPS sobre las cuales ejerce la vigilancia y control de manera irregular y esporádica, omitiendo las acciones sobre las entidades administradoras de planes de beneficios; Respecto del cumplimiento del Sistema Único de Habilitación en la mayoría de zonas evaluadas este se limita al registro de la existencia de prestadores y visitas en atención de quejas, sin contar con procesos orientados a realizar evaluaciones sistemáticas. En síntesis la función de IVC de las redes de salud de los territorios evaluados no ha sido realizada a plenitud y por lo mismo no existen programas para acompañar la gestión en calidad en la atención para NNA.

\section{DISCUSION}

El concepto de calidad, que ha evolucionado de un esquema de control a un esquema de garantía tiene al menos seis posibilidades de abordaje:

1. Considerar la calidad como un conjunto de atributos, que no son más que características positivas y deseables para calificar la atención en salud la cual resultara adecuada cuando dichos atributos están presentes en un nivel deseado.

2. Entender la calidad desde la satisfacción de las necesidades de demanda y oferta, evaluando los procesos, los resultados y el impacto de las acciones. 
3. Enfocarse en la gradualidad, considerando los recursos disponibles para los diferentes procesos de atención en salud.

4. Encaminarse a optimizar los beneficios y por ende la eficiencia y eficacia que deben acompañar la calidad de la atención.

5. Orientarse a la minimización de riesgos mediante la administración de los mismos y la implementación de modelos de atención basados en la prevención.

6. Incluir técnicas basadas en la subjetividad o calidad sentida (17).

Para el trabajo en calidad se requiere de indicadores que no son más que inductores de acción, es decir que son expresiones cuantitativas o cualitativas que corresponden al resultado de una medición que, al contrastarlos con un estándar previamente establecido, determinan si el resultado se encuentra en rangos de normalidad o calidad esperada, o si, por el contrario, se está por fuera de dichos rangos y resulta por ende necesaria la toma de acciones inmediatas tendientes a la corrección de dicha desviación (18).

En este contexto en Colombia no se encontraron antecedentes de estudios que evalúen la gestión de la calidad en salud en programas de infancia específicamente, sin embargo existen algunos reportes relacionados, como el estudio realizado por la veeduría distrital acerca de la situación de los niños y las niñas en Bogotá 2007-2008. Este estudio ofrece un diagnóstico de la población infantil desde la perspectiva de los derechos, postulando que a favor de los NNA se debe posibilitar el disfrute del más alto nivel posible de salud y servicios para el tratamiento de las enfermedades y rehabilitación de la salud y por tanto deberían incluirse al futuro indicadores trazadores que permitan medir la garantía de calidad de los servicios (19).

En el ámbito internacional hay diferentes estudios que evalúan la calidad de la atención de los servicios de salud prestados a la población menor de 18 años, así por ejemplo, en Argentina se realizó una evaluación de la calidad del sistema de registro y tratamiento de las enfermedades prevalentes de la infancia (20), en México se realizó uno de detección y análisis de los factores inhibidores de la calidad en los servicios de salud (21), en España se trabajó acerca del cuidado de los adolescentes en los hospitales (22); Otros se han interesado por la satisfacción del paciente como indicador de calidad $(23,24)$ o por la evaluación de herramientas para medir la calidad de la atención (25); Incluso hay evaluaciones de las acciones administrativas sobre los sistemas de salud en lo referente a la 
mejora de la calidad de actividades preventivas y de promoción de la salud (26), pero no se encontraron estudios dirigidos a la evaluación de calidad de programas específicos de atención a población infantil por parte de gobiernos locales, por lo que un estudio de este tipo se convierte en línea base para futuras mediciones de la gestión de la calidad de los programas.

Una línea base que para nuestro caso se evidencia como deficiente, ya que no existe una gestión de la calidad para programas específicos sino apenas acciones aisladas de inspección, vigilancia y control del sistema en forma general, y al no realizar un seguimiento regular a los sistemas de calidad de los agentes del sistema, no se están verificando los requisitos para el proceso de habilitación y no se están verificando que los mismos ejecuten acciones de mejoramiento continuo en cuanto a la garantía de la calidad de la atención en salud. Esta situación repercute directamente en el servicio que reciben los beneficiarios del Sistema General de Seguridad Social en Salud.

La explicación presentada por estos gobiernos también resulta insatisfactoria por cuanto evidencia una excesiva dependencia del nivel central para el desarrollo de sus funciones, contradiciendo los principios de descentralización y autonomía administrativa y poca creatividad a la hora de utilizar estrategias modernas de gestión.

Mención especial merece el hecho de que el sector salud ha sufrido con intensidad los procesos de flexibilización laboral (27) por lo que con frecuencia los colaboradores de los gobiernos locales viven situaciones de inestabilidad y malestar laboral, lo que limita la calidad de los procesos adelantados.

En síntesis, no hay un nivel suficiente de gestión de la calidad en salud en los gobiernos locales para los programas de atención a NNA y su esfuerzo se limita al cumplimiento de la normatividad general del SGSSS con acciones aisladas producto al parecer de la limitación en los recursos, por lo que se sigue en deuda, en materia de salud, con la población infantil colombiana.

Por lo anterior, se sugiere que el accionar de los gobiernos locales debe enfatizar las acciones dirigidas a lograr que las instituciones a su cargo presten asistencia en salud bajo los parámetros de calidad establecidos en 
la normatividad vigente y dado que la función de los entes territoriales en materia de calidad va desde la planeación pasando por la rectoría y el control de la calidad de los prestadores, se hace inaplazable la implementación de programas de gestión de la calidad antes que nuevos programas de atención.

Dichos programas deben contar con recursos económicos que permitan solventar las limitaciones de recurso humano y con estrategias de georreferenciación que permitan hacer frente a las dificultades de dispersión de entes para de este modo reactivar metodologías de mejoramiento continuo que permitan elevar los niveles de calidad de la atención y con ellos los niveles de salud de la población, en este sentido el ajuste a la reforma, aprobado recientemente, propone entre otros un modelo de competencias de los diferentes niveles de la administración pública, un plan decenal de salud pública, programas de coordinación intersectorial, la creación del observatorio nacional de salud, la obligación de atención preferente para NNA, la construcción de indicadores de salud específicos y la desconcentración y descentralización de algunas de las funciones de la superintendencia nacional de salud, aspectos acerca de los cuales hay que esperar su impacto sobre la línea de base aquí construida

Agradecimientos: A los equipos de trabajo que realizaron las visitas a los diferentes territorios y al a superintendencia nacional de salud por el acceso a los informes y soportes.

\section{REFERENCIAS}

1. Departamento Administrativo Nacional de Estadística. Información estadística. Censos y demografía. Series y proyecciones. Estadísticas vitales. Estadísticas demográficas. Bogotá: DANE; 2010.

2. Carmona-Fonseca J. Cambios demográficos y epidemiológicos en Colombia durante el siglo XX. Biomédica. 2005; 25 (4):464-480.

3. Ruelas E, Cocho G, Villegas M. Las ciencias de la complejidad y la innovación médica. En complejidad, sistemas de salud y calidad. Bogotá: Grama editora; 2006.

4. Hernández-Montoya W. Gómez P. Identificación de familias prioritarias para la atención en salud en una Comunidad urbano - marginal de la ciudad de Cartago. Rev costarric salud pública. 2000; 9(16):65-75.

5. Spinak E. Indicadores cienciometricos. Ci Inf 1998; 27(2):141-148.

6. Colombia. Congreso. Ley 100 de 1993, diciembre 23, por la cual se crea el sistema de seguridad social integral y se dictan otras disposiciones. Bogotá: El Congreso; 1993, art. 153.

7. Sarmiento A. La institucionalidad social en Colombia: la búsqueda de una descentralización con centro. Bogotá: Editorial Naciones Unidas; 2004.

8. UNICEF. [Internet]. Disponible en: http://www.unicef.org/about/who/index_introduction. html. Consultado Noviembre 2010. 
9. República de Colombia. Constitución Política de Colombia, 1991. Artículo 44. 10. Ley 1098 de 2006. República de Colombia. Diario Oficial 46446:1-28.

11. Ley 1122 de 2007. Artículo 40, República de Colombia. Diario Oficial 46506:60-68.

12. García-Ubaque J. Morales O. [Internet]. Situación de los programas de atención en salud para la población de niños, niñas, adolescentes, jóvenes y mujeres desplazadas en 21 departamentos y 20 capitales de departamento en Colombia. Comunicados de prensa Superintendencia Nacional de Salud. Mayo 2009. Disponible en:http://www. supersalud.gov.co/supersalud/ Link Click.aspx?fileticket=Tu\%2Bs7jYvwHs\%3D\&ta bid=59 \&mid=1289. Consultado Junio 2010.

13. Muñoz F, López-Acuña D, Halverson P, Macedo C, Hanna W, Larrieu M., et al. Las funciones esenciales de la salud pública: un tema emergente en las reformas del sector de la salud. Pan Am. J. Public Health. 2000; 8(1-2):126-134.

14. UNICEF. Vulnerabilidad de los niños y las niñas colombianos. [Internet]. Disponible en: http://www.unicef.org.co/retorno/03-contexto-vuln.htm. Consultado Junio 2010.

15. Weiss K. Wagner R. Performance Measurement Through Audit, Feedback, and Profiling as Tools for Improving Clinical Care. Chest. Ago. 2000; 118 (Suplemento):53S-58S.

16. Decreto 1011 de 2006. República de Colombia. Diario Oficial 46230:35-40 Articulo 3

17. Bautista A, García-Ubaque JC. Las infecciones adquiridas en el hospital en el contexto de salud pública. En: Malagón-Londoño G. Álvarez C. Infecciones hospitalarias. Bogota Colombia, Editorial Médica Panamericana; 2010.

18. Pérez $M$, Pérez $R$, Moreno $M$, Llanes $M$ Procedimiento para determinar y evaluar estándares de calidad. Observatorio de la Economía Latinoamericana 2009; 121:1-9. Disponible en: http//www.eumed.net/cursecon/ecolat/cu/2009/dcpf.htm. Consultado Noviembre 2010.

19. Rodríguez -Fernández J, Martínez J, Monserrat-Capella D, Sainz A, Cebrian M, GarciaArtiaga C, Muñoz V. Utilización de la opinión de los pacientes para la mejora: 5 años de seguimiento (2001-2005). Revista de Calidad Asistencial. 2008; 23(2):5764.

20. Zimmer M, Villagrán E, Valdivieso M, Mosqueira R, Contreras N. Evaluación de la calidad de la asistencia prestada a niños menores de 6 años en centros de salud en SaltaCapital (Argentina). Revista de Calidad Asistencial. 2006; 21(3):150-154.

21. Martínez-Ramírez A, Chávez-Ramírez S, Cambero-González E, Ortega-Larios E, Rojas Z. Detección y análisis de los factores inhibidores de la calidad en los servicios de salud (FICSS). Revista de Calidad Asistencial. 2008; 23(5):205-215.

22. Ullan A, González R. El cuidado de los adolescentes en los hospitales españoles: los pacientes invisibles. Revista de Calidad Asistencial. 2010; 25(3):146-152.

23. Álvarez M. Evaluación de los criterios satisfacción del cliente/paciente y satisfacción del personal/profesional. Revista de Calidad Asistencial. 2007; 22(5): 227-233.

24. Aymerich M, Berra S, Guillamon I, Herdman M, Alonso J, Ravens-Sieberer U, Rajmil L. Desarrollo de la versión en español del KIDSCREEN, un cuestionario de calidad de vida para la población infantil y adolescente. Gaceta Sanitaria. 2005; 19(2): 93-102.

25. Sardón O, Morera G, Herdman M, Moreno A, Pérez E, Detmar S, Fekkes M, Rajmil L, Versión española del TAPQOL: Calidad de vida relacionada con la salud en niños de 3 meses a 5 años. Anales de Pediatría. 2008; 68 (5):420-424.

26. Escribano M, Moreno J, Arias G, Herranz J, Gomis R. Evaluación y mejora de la calidad del Programa de Actividades Preventivas y Promoción de la Salud. Atención Primaria. 2006; 38(5):283-287.

27. Ley 1438 de 2011. República de Colombia. Diario Oficial 47957:1-14. 